Najib Mahdou • Sanae Moussaoui •

Moutu Abdou Salam Moutui

\title{
Erratum: When every finitely projective ideal is projective
}

Published online: 28 February 2022

(C) The Indian National Science Academy 2022

\section{Correction to: Indian Journal of Pure and Applied Mathematics https://doi.org/10.1007/s13226-021-00148-y}

In Section 2:

There is a problem in the proof of Lemma 2.4 of the previous version. Indeed, h' is not an S-homomorphism. So, we remove Lemma 2.4 and therefore, assertion 1 of Theorem 2.5, Remark 2.5 and Proposition 2.7 were also removed, as Lemma 2.4 were involved in these results.

In Section 3:

The assertion 2 of Theorem 3.1 was replaced by Remark 3.6 and therefore Example 3.9 which was using assertion 2 of Theorem 3.1 was removed.

In Section 4:

In assertion 2 of Theorem 4.1, we have added the condition $\mathrm{f}(\mathrm{I}) \mathrm{J}=0$ in order to get that the homomorphism $\pi$ ' used in the proof of Lemma 4.3 be well defined.

The original article can be found online at https://doi.org/10.1007/s13226-021-00148-y.

N. Mahdou $\cdot$ S. Moussaoui

Laboratory of Modeling and Mathematical Structures, Department of Mathematics, Faculty of Science and Technology of Fez, University S.M. Ben Abdellah Fez, Box 2202, Fez, Morocco

e-mail: mahdou@hotmail.com

S. Moussaoui

e-mail: sanaemoussaoui16@gmail.com

M. A. S. Moutui $(\varangle)$

Division of Science, Technology, and Mathematics, American University of Afghanistan, Kabul, Afghanistan e-mail: mmoutui@auaf.edu.af 